\title{
Ameliorated Human Anatomical Specimen Fixative Solution and Its Application
}

\author{
Wei Dequan, Shang Qi \\ Zaozhuang Vocational College of Science \& Technology,Tengzhou,Shandong,277500,China
}

Keywords: Anatomic specimen;fixative solution;Application analysis;

\begin{abstract}
Purpose:To introduce and analyze the application mode of the ameliorated human anatomical specimen fixative solution. Methods:The ameliorated fixative solution of human anatomical specimen was selected, and then the infusion, preservation solution infiltration and gradually drying work were done. The specimen is preserved in time, and the final purpose is to compare and analyze with the other preserved specimens.Result:By applying the human anatomical specimen to human anatomical specimen fixative solution, the elasticity, the color and the natural form of the fixative solution can be fully promoted, the toxicity and irritation of the fixative solution are also relatively small. There are characteristics of environmental protection, convenience and economy, which promotes the save quality. Conclusion:In the specific application process, the use of the ameliorated fixative solution of human anatomical specimen can fully satisfy the basic preservation needs of human anatomy specimens. And it is very beneficial for the improvement of experimental teaching environment, and has important popularization and application value.

Human anatomical science belongs to a kind of morphological science. It can be applied to the application of various anatomical specimens in medical anatomy. Therefore, in order to effectively meet the needs of the experiment, the use of fixative solution in human anatomy has been a high frequency [1],It is widely used in clinical medicine. One of the more traditional fixative solution is a formalin, which has obvious advantages in bactericidal and antiseptic, and the price is relatively easy to get. However, the shortcomings of the using process is more obvious. It has a strong irritation and can produce a serious harm in clinical[2]. With the gradual deepening of various studies, researchers have found that formaldehyde is more likely to cause cancer and can lead to a variety of cancers. Including the induction of gene mutation. Therefore, the clinical use of formaldehyde is being gradually reduced and a new type of fixative solution is being explored. At the same time, it is set as an efficient and low-toxicity target in the study and the fixative solution of the human anatomical specimen is improved.
\end{abstract}

\section{Research materials and research methods}

\subsection{Research materials}

The formulation of the study includes four recipes. The first formulation is $15000 \mathrm{ml}$ of water, $750 \mathrm{ml}$ of formaldehyde, $15 \mathrm{~g}$ of thymol, $300 \mathrm{~g}$ of potassium nitrate and $450 \mathrm{~g}$ of potassium acetate. The second formula is $1600 \mathrm{ml}$ eighty degree hot water, $160 \mathrm{~g}$ sodium chloride, $1600 \mathrm{ml}$ glycerol, $250 \mathrm{ml} \mathrm{90 \%} \mathrm{ethanol.The} \mathrm{third} \mathrm{formulation} \mathrm{is} \mathrm{six} \mathrm{hundred} \mathrm{milliliters} \mathrm{of} \mathrm{eighty} \mathrm{degrees} \mathrm{hot} \mathrm{water,} 200$ grams of sodium chloride, 1600 milliliters of glycerol, and 200 milliliters of 95 percent ethanol.The fourth formulation is $600 \mathrm{ml}$ of eighty degree hot water, $200 \mathrm{~g}$ of sodium chloride, $400 \mathrm{ml}$ of glycerol, and $200 \mathrm{ml}$ of $95 \%$ ethanol. In addition to the first recipe are required to use a few fragrances.

\subsection{Research methods}

\subsubsection{Analysis of pretreatment process}

First, anticorrosive pretreatment for fresh specimens of non-corrosive bodies, Which Includes shaving and cleaning. Then, according to the requirements of anatomy, we take a flat posture and take a prone position,then cut the femoral artery in the inguinal position of the body, the T-tube insertion, and finally effective fixation. 


\subsubsection{Perfusion}

Choose different ways of perfusion according to the different formula. Make full use of the T-tube in the first recipe, one-time injection of $10,000 \mathrm{ml}$ of fixing solution, and formaldehyde is used in the choice of preservative. Potassium nitrate and potassium acetate with the role of color retention and preservation, the role of thymol is to reduce the occurrence of mold. After that, Put it in the freezer box and control the temperature at about five degrees. After ten days of freezing, 1000 $\mathrm{ml}$ of $50 \%$ ethanol is injected and $100 \mathrm{ml}$ of ammonia was added. After a further ten days of freezing, add $1000 \mathrm{ml}$ of $50 \%$ ethanol and $100 \mathrm{ml}$ of ammonia. After another ten days, $1000 \mathrm{ml}$ of $70 \%$ ethanol was injected, and $120 \mathrm{ml}$ of ammonia water was injected. Ten days later, $90 \%$ ethanol was injected, and $100 \mathrm{ml}$ of ammonia water was injected. Under the joint action of both, it can reduce the stimulation odor caused by the presence of formaldehyde, and also have the effect of anticorrosion.

At the same time in the second formula, set a total of three perfusion, each interval is three days, the total perfusion was set at $12500 \mathrm{ml}$. In the third formula, the same three perfusion was performed, and the total amount of perfusion was set at $12000 \mathrm{ml}$. In the fourth formulation, perfusion was performed twice with a perfusion interval of five days each and the total amount of perfusion set at about $12000 \mathrm{ml}$. At the same time, the total amount of perfusion was adjusted according to the difference of the average corpse's average mass.

\subsubsection{Preservation}

After completion of the body perfusion and specimen preparation,the plastic bag is used for sealing and packing, while it is infiltrated for three weeks to four weeks. The infiltration of fixative solution is fully carried out inside the body tissue, and the specimen is made. Then using stainless steel brackets and wooden racks fully fixed, and placed it in the room. This can effectively reduce the dust contamination. Finally, the specimens were used effectively and the specimens were covered with cloth after use.

\subsection{Formulation of Eco - preservation Solution and Analysis of Its Physical and Chemical Properties}

The main components of environmental preservation solution are osmosis regulator, chitosan and other natural antibacterial agents, fat hydrolysis inhibitors, food or leather preservatives and metal ion complexing agents. The operation mechanism in the experimental process is to make full use of the conditions for protein denaturation and enzyme inactivation, food, metal ion complexing agents, leather anti-corrosion and natural antibacterial agents. The components of leather preservatives include heterocyclic and sodium diacetate. The purpose is to promote enzyme inactivation and protein solidification, and fully immobilize the specimen and promote the effective occurrence of its preservation. At the same time using the principle of semipermeable membrane penetration to make osmotic pressure regulator fully integrated. While maintaining the preservation solution and the sample solution in an isotonic environment, the purpose is to prevent the occurrence of the specimen over-hardening and swelling. In the protein and fat hydrolysis and degradation products composed of polyols, nitro salts and amino acids, adding a certain amount of protein and fat hydrolysis inhibitors, which is make up by polyols, amino acid salts and nitrate, etc.,. Such a way of operation can inhibit the hydrolysis of protein and fat, fully degrade the mobile equilibrium, and ultimately promote the good effect of re immobilization, moisture retention and preservation.

It needs to be fully preserved and compared during the first use of environmental solution. Two sets of specimens are needed for the preservation of the specimen,one for environmental preservation solution, and the other for formaldehyde preservation solution. During the process of application, decay, shrinkage, moldy, defatting and discoloration appeared. At the same time, there would be multiple observation and re extraction during the experiment. However, it can still effectively preserve the toughness and shape of the object, and there is no embrittlement and breakage. The use of formaldehyde preservation solution has the above good effect, and it does not have an irritating taste. It has important value in clinical application and practical research. Through a large number of studies, we can see that the clinical irritation of the environmental preservation solution is relatively small compared with the formaldehyde preservation solution. The replacement 
of formaldehyde solution into environmental solution has a certain maneuverability and positive significance in clinical.

\section{Result}

\subsection{Effect of using}

The specific effect of using the fixation solution for human anatomy and the use of formalin are shown in Table 1 below.

Table 1 Comparison and analysis of the application effect of the ameliorated human anatomy specimen fixative solution and the formalin solution

\begin{tabular}{cccc}
\hline Project & & $\begin{array}{c}\text { Ameliorated } \\
\text { human anatomy } \\
\text { specimen fixative } \\
\text { solution }\end{array}$ & Formalin solution \\
\hline Human & Irritations & Slight & Strong \\
body & Poisons & Slight & Serious \\
Corrupt and & No & No \\
Specimen & mouldy & Well & Commonly \\
& Form and color & Well & Poor \\
\hline
\end{tabular}

Compared with the traditional method, the ameliorated fixative solution of the human anatomy specimen can be effectively guaranteed the natural form, color and elasticity of the specimen. At the same time, it also has relatively small irritation and toxicity, and it is also portable and low economic cost, which can effectively guarantee the quality of the specimen.

\subsection{Analysis of formaldehyde preservation solution and its harm to human body}

The use of formaldehyde in the experiment can play an effective role in sterilization and antibacterial. It has good permeability and stretching effect, which can effectively protect lipid-like lipids in the tissue and the full fixed and preserved specimens without shrinkage, and promote the production of non-degreasing decalcification, ultimately undistorted, fully enhanced the understanding of human tissues and organs. At the same time, the formaldehyde prices are relatively low, the configuration of preservation solution is relatively simple and easy to obtain. Long-term preservation of specimens can be achieved. It is also not easy to appear deterioration, decay and moldy phenomenon, and we can fully realize the long-term use of specimens. However, at room temperature in the use of state, the formaldehyde in the preservation solution is relatively volatile, which can increase the body's acute toxicity. Clinically, it can bring respiratory, skin and conjunctival stimulation, eventually lead to tearing, eye pain and dermatitis. Clinically, long-term exposure to formaldehyde at a concentration of $0.45 \mathrm{mg} / \mathrm{m} 3$ will result in some increase in chronic respiratory disease. If the inhalation of formaldehyde in a relatively high concentration, it will bring a relatively strong mutagenic effects, bronchospasm and larynx and pulmonary edema, wheezing and respiratory failure and the eventual death may occur. The criterion for determining high concentration of formaldehyde is $60-120 \mathrm{mg} / \mathrm{m} 3$ and above. Meanwhile, the use of formaldehyde will also bring a strong mutagenic effect. Under long-term exposure, it can lead to multiple organ and multiple system damage, and ultimately improve the incidence of cancer in contact population and is a type of carcinogen.

\section{Discussion}

In the process of human anatomy, there are many uses for human anatomy specimens. For the preservation of specimens, the traditional way of preservation is to use formalin solution[3].The use of this method has effective anticorrosion effect, strong bactericidal and economic performance, and has a wide range of use. However, during the specific time of application, if the specimen is infused, infiltrated and preserved by formalin, it may bring strong toxicity and irritation, which will adversely affect the health of the user. The research of foreign scholars shows that formaldehyde 
contained in the formalin solution is a carcinogen, if it was used for a long time, it may lead to a variety of carcinogenic substances, gene also has some damage, including long-term mutation behavior under the influence of its role[4].In order to minimize the adverse effects of formaldehyde, the relevant experts and scholars are actively studying the new fixative solution. The development of the fixative solution has the characteristics of high efficiency, low toxicity and environmental protection.

In this paper, we have studied the fixative solution of human anatomy fluid, the main component of which is glycerin and with strong hygroscopicity and hydrophilicity in the process of use. By mixing ethanol with water, the stability of the solution can be effectively guaranteed. For the use of human specimens may lead to the production of drying phenomenon, the use of this component can effectively reduce this phenomenon and prevention, while effectively ensuring the flexibility of the phenomenon.The application of it can effectively guarantee the stability of the solution. It can also effectively avoid the possible occurrence of loss and volatilization, and has a significant promotion effect on the enhancement of anti-corrosion ability. Through the use of glycerol, formaldehyde evaporation can be effectively controlled. The application of this phenomenon can effectively improve the operating environment of the experiment, and improve the effective control of irritation and toxicity.

Through the above studies, it can be seen that it has obvious advantages to fully exert the role of the ameliorated fixative solution of human anatomy specimen in the process of specific application. In the preservation effect, it can achieve the ideal effect, at the same time, it is relatively slight in irritation and harmfulness. It can effectively promote the full progress of the experiment, enhance the safety and efficiency of the experiment, and can achieve the ideal goal of human anatomy.

\section{Reference}

[1] Chai Hua.Application of ameliorated human anatomical specimen fixative solution[J].Journal of Medicine, 2016(6):00280-00280.

[2] Liu Dongqiang. Study on Reducing Formaldehyde Smell of Human Anatomical Experimental Teaching Specimens[J].Journal of Reading Digest: Mid, 2016(18).

[3] Fan Jiangjian. Application of Plasticized Specimens in the Making of Human Anatomy Specimens[J].Journal of Medicine front,2014(11):128-129.

[4] Dong Difeng,Yu Ping,Yin Lizhu.,etc. Human Anatomy Liver Specimen Making Method[J].Journal of Medical Aesthetics and beauty, 2015, 24(6):894-895. 